\title{
ON REAL INVARIANT SUBSPACES OF BOUNDED OPERATORS WITH COMPACT IMAGINARY PART
}

\author{
VICTOR I. LOMONOSOV
}

(Communicated by Paul S. Muhly)

\begin{abstract}
In this paper we show the existence of real invariant subspaces for compact perturbations of selfadjoint operators.
\end{abstract}

\section{INTRODUCTION}

One of the most difficult questions in the theory of invariant subspaces is the problem of the existence of invariant subspaces for a compact perturbation of a selfadjoint operator. Livsič solved this problem for nuclear perturbations, Sahnovič for Hilbert-Schmidt perturbations, and Gohberg and Kreinn, Macaev, and Schwartz for the perturbation being in the Schatten's class $\mathscr{C}^{p}$. Macaev proved the spectral separation theorem for a perturbation in the class $\mathscr{S}_{w}$, and moreover, if the operator does not belong to the class $\mathscr{S}_{w}$ then its spectrum cannot be separated in general. (Recall that the operator $K$ belongs to the class $\mathscr{S}_{w}$ if its $s$-numbers satisfy $\sum_{j=1}^{\infty} s_{j}(K) /(2 j-1)<\infty$.) However, there is still hope to prove the existence of invariant subspaces for every compact perturbation of a selfadjoint operator. In this paper we do so for real subspaces.

\section{THE RESULT}

Let $\mathscr{H}$ be a complex Hilbert space. A subset of $\mathscr{H}$ is called a real subspace if it is closed under vector addition and multiplication by real scalars. Let $A$ be a bounded linear operator on $\mathscr{H}$, and suppose that $A=R+K$, where $R$ is a selfadjoint operator and $K$ is a compact operator. In this paper we prove the following result.

Theorem 1. Let $A$ be a bounded linear operator on $\mathscr{H}$, and suppose that $A=$ $R+K$, where $R$ is selfadjoint and $K$ is compact. Then $A$ has a nontrivial closed real invariant subspace.

In order to prove the theorem we need the following well-known fact from the theory of invariant subspaces. Recall that an operator $A$ on the space $\mathscr{H}$ is said to be quasi-triangular if there exists a sequence $\left\{Q_{n}\right\}_{n=1}^{\infty}$ of finite rank

Received by the editors December 10, 1990 .

1980 Mathematics Subject Classification (1985 Revision). Primary 47A15.

Key words and phrases. Compact perturbation, real invariant subspace. 
orthogonal projections strongly converging to $I$ such that

$$
\lim _{n \rightarrow \infty}\left\|\left(I-Q_{n}\right) A Q_{n}\right\|=0 .
$$

Lemma 1. If $A$ is quasi-triangular then there exists a sequence $\left\{P_{n}\right\}_{n=1}^{\infty}$ of finite rank orthogonal projections that converges weakly to a selfadjoint operator $S$ other than 0 or I with the property that

$$
\lim _{n \rightarrow \infty}\left\|\left(I-P_{n}\right) A P_{n}\right\|=0 .
$$

(For the proof of the lemma see, e.g., [1, p. 85].)

Let us make several remarks.

(1) The operator $A$ from the theorem is obviously quasi-triangular. Indeed, the spectral theorem implies that a selfadjoint operator is quasi-triangular, and a compact perturbation preserves this property.

(2) Any positive power $A^{\ell}$ can be represented as $A^{\ell}=R_{\ell}+K_{\ell}$, where $R_{\ell}$ is selfadjoint and $K_{\ell}$ is compact.

(3) For any positive integer $\ell$ the following assertion holds:

$$
\lim _{n \rightarrow \infty}\left\|\left(I-P_{n}\right) A^{\ell} P_{n}\right\|=0 .
$$

Note that this formula means that an almost invariant subspace of $A$ must be almost invariant for any positive power of $A$. Indeed, the case $\ell=1$ is covered by lemma, and for $\ell>1$ we use induction,

$$
\begin{aligned}
\left\|\left(I-P_{n}\right) A^{\ell} P_{n}\right\| & =\left\|\left(I-P_{n}\right) A^{\ell-1}\left(I-P_{n}+P_{n}\right) A P_{n}\right\| \\
& \leq\left\|\left(I-P_{n}\right) A^{\ell-1} P_{n} A P_{n}\right\|+\left\|\left(I-P_{n}\right) A^{l-1}\left(I-P_{n}\right) A P_{n}\right\| \\
& \leq C_{1}\left\|\left(I-P_{n}\right) A^{\ell-1} P_{n}\right\|+C_{2}\left\|\left(I-P_{n}\right) A P_{n}\right\| .
\end{aligned}
$$

Returning to the proof of the theorem. We have

$$
\begin{aligned}
A^{\ell} S-S A^{\ell} S= & (\text { weak })-\lim _{n \rightarrow \infty}\left(A^{\ell} P_{n}-S A^{\ell} S\right) \\
= & (\text { weak })-\lim _{n \rightarrow \infty}\left(P_{n} R_{\ell} P_{n}-S R_{\ell} S\right) \\
& +(\text { weak })-\lim _{n \rightarrow \infty}\left(P_{n} K_{\ell} P_{n}-S K_{\ell} S\right) .
\end{aligned}
$$

The second term of the last sum converges to zero thanks to the compactness of the operator $K_{\ell}$, and the first term converges to a selfadjoint operator $T_{\ell}$. Therefore we have $(I-S) A^{\ell} S=T_{\ell}$.

Let $x$ be a vector in $\mathscr{H}$ such that both $S x \neq 0$ and $(I-S) x \neq 0$. Then for any positive integer $\ell$ we have

$$
\operatorname{Im}\left\langle A^{\ell} S x,(I-S) x\right\rangle=\operatorname{Im}\left\langle T_{\ell} x, x\right\rangle=0 .
$$

Consider the real closed subspace $L$ of $\mathscr{H}$ spanned by the vectors $\left\{A^{\ell} S x\right\}$. Obviously this subspace does not contain the vector $i(I-S) x$ and thus $L$ is a nontrivial invariant subspace of the operator $A$. Thus the proof of the theorem is complete.

Note that the subspaces $L \cap i L$ and $\overline{\operatorname{span}}(L, i L)$ are complex invariant subspaces of the operator $A$. Assuming that the operator $A$ does not have invariant subspaces, we have $L \cap i L=\{0\}$ and $\overline{\operatorname{span}}(L, i L)=\mathscr{H}$.

If, in addition, $L$ and $i L$ were orthogonal then the operator $A$ would have a real form. 
Therefore the lack of existence of invariant subspaces for $A$ gives the reward of a real form for $A$.

The preceding argument leads to the following question: Does there exist real invariant subspaces for an arbitrary bounded operator on a complex Hilbert or Banach space?

\section{REFERENCES}

1. H. Radjavi and P. Rosenthal, Invariant subspaces. Springer-Verlag, New York, Heidelberg, Berlin 1973.

Department of Mathematics, Kent State University, Kent, Ohio 44242 